\title{
A new contact damping model of joint interfaces with simulation Jingfang Shen ${ }^{1,2, a}$, Yue Liu ${ }^{2, b}$, Sha $\mathrm{Xu}^{2, \mathrm{~b}}$, Wenwei Liu ${ }^{1, \mathrm{c}}$ and Jiajun Yang ${ }^{1, \mathrm{c}}$ \\ ${ }^{1}$ School of Mechanical Science and Engineering, Huazhong University of Science and Technology, Wuhan, 430074, China; \\ ${ }^{2}$ School of Science, Huazhong Agricultural University, Wuhan,430070, China. \\ a shenjingfang16@126.com, 'YULIU_HZAU@126.com, cyjjllar@163.com
}

Keywords: Normal contact damping, Fractal model, Equal radius, Numerical simulation.

\begin{abstract}
Modifying joint interfaces' contact model of two spheres makes contact model more universal. On the basis of contact theory, energy dissipation mechanism and domain expansion factor's effect, the elastoplastic fractal model of contact damping on two spheres is constructed. Numerical simulation results reveal that there is an apparent concave nonlinear relation between normal damping and load on two spheres' joint interfaces, when fractal dimension is in range of $1.2 \sim$ 1.4. Normal damping increases with normal load. Instead, its relation is convex nonlinear,when fractal dimension is in range of 1.6 1.8. It decreases with the increasing of normal load. When fractal dimension is 1.4, normal damping decreases with the increasing of fractal characteristic length parameters, when fractal dimension is 1.6, it increases with fractal characteristic length parameters.
\end{abstract}

\section{Introduction}

In microscopic domain, two or more than two objects realizing the problem of force transmission through topical boundary contact with each other is called contact problem. Exactly speaking, all mechanical contact on friction pairs' surfaces is rough surface contact in different degree of roughness. General contact surfaces are not smooth. They are always made up of many irregular convex peaks and valleys. Actual contact only occurs on friction surfaces. Recent research indicates that real rough surfaces is composed of surface morphology with distinct scale levels of fractal traits or multi-scale features ${ }^{[1]}$. Contacting on rough surfaces can markedly impact friction, wear, lubrication, sealing and heat transfer. So it is an essential part of the study of tribology. Hertz first put forward the force distribution of two elastic spheres in extrusion. Li ${ }^{[2]}$ gave a "solid-gap-solid" contact model. Zhang ${ }^{[3]}$ proposed a modified MB fractal model and researched on mechanism of contact normal damping dissipating energy. Some scholars ${ }^{[4-7]}$ indicated that considering contact mechanism of rough surfaces as a single complete elastic or complete plastic deformation, or elastic and plastic deformation simultaneously has certain deficiencies and simplified two rough surface as a rough surface and a rigid plane. In this paper, we modify two surfaces as two spheres with equal radius and fully consider the elastic, elastic-plastic, plastic contact deformation mechanism based on normal damping energy dissipation mechanism and normal damping theory on two spheres' surface. This model will be more general and comprehensive. Discussing the relation of damping $C_{n}^{*}$, total normal load $P^{*}$, fractal characteristic length parameters $G^{*}$, and doing numerical simulation.

\section{Modeling of Joint Surface}

Wang and Komvopoulos ${ }^{[8]}$ introduced micro contact distribution domain extension factor $\psi$, so the distribution function of micro contact on cross-sectional area is

$$
n\left(a^{\prime}\right)=\frac{D}{2} \psi^{\frac{2-D}{2}} a_{l}^{\prime^{\frac{D}{2}}} a^{\prime^{-\frac{D+2}{2}}} \quad 0<a^{\prime} \leq a_{l}{ }^{\prime}
$$

where $D$ is fractal dimension on surface, $a^{\prime}$ is cross-sectional area of micro contact, $a_{l}{ }^{\prime}$ is maximum cross-sectional area of micro contact.

When curvature radii of a single micro convex body on two joint surfaces are equal, the formula of 
$P_{e}$ is given

$$
P_{e}=\frac{2 \sqrt{2}}{3} E^{*} R^{\frac{1}{2}} \delta^{\frac{3}{2}}
$$

The contact area on plastic contact point and the normal contact load $P_{p}$ are

$$
P_{p}=k \sigma_{y} a^{\prime}
$$

where $\delta$ is normal form variable, $E^{*}$ is complex elastic modulus, $k$ is coefficient of hardness and yield strength of soft materials, $\sigma_{y}$ is soft material yield strength.

\section{The Elastic and Plastic Strain Energy}

The elastic strain energy of a single micro convex body is

$$
\omega_{e}=\int_{0}^{\delta} P_{e} d \delta=\int_{0}^{\delta} \frac{2 \sqrt{2}}{3} E^{*} R^{\frac{1}{2}} \delta^{\frac{3}{2}} d \delta=\frac{4 \sqrt{2}}{15} E^{*} R^{\frac{1}{2}} \delta^{\frac{5}{2}}
$$

So the elastic strain energy produced in the contact area is

$$
W_{e}=\int_{a_{c}}^{a^{\prime}} \omega_{e} n\left(a^{\prime}\right) d d=\frac{E^{*} D \pi^{\frac{3}{2}} G^{2(D-1)}}{15(5-3 D)} \psi^{\frac{2-D}{2}}\left(a_{l}{ }^{\frac{5-2 D}{2}}-a_{l}{ }^{\frac{D}{2}} a_{c}{ }^{\frac{5-3 D}{2}}\right)
$$

The plastic strain energy of a single convex body is

$$
\omega_{e}=\int_{0}^{\delta} P_{p} d \delta=\int_{0}^{\delta} k \sigma_{y} a d \delta=k \sigma_{y} a^{\prime} \delta
$$

So the plastic strain energy produced in the contact area is

$$
W_{p}=\int_{0}^{a_{c}} \omega_{p} n\left(a^{\prime}\right) d a^{\prime}=\frac{k \sigma_{y} D G^{D-1}}{2(2-D)} \psi^{\frac{2-D}{2}} a_{l^{\prime 2}} a_{c}{ }^{\prime 2-D}
$$

The damping loss factor on the joint surface is

$$
\eta=\frac{W_{p}}{W_{e}}=\frac{15 k \sigma_{y}(5-3 D) a_{c}{ }^{12-D}}{2 E^{*} G^{D-1}(2-D)\left(a_{l^{1^{\prime}}}{ }^{\frac{-3 D}{2}}-a_{c}{ }^{\frac{5-3 D}{2}}\right)}
$$

The normal contact stiffness of joint surface is

$$
K_{n}=\frac{D E^{*}}{\sqrt{\pi}(1-D)} \psi^{\frac{2-D}{2}} a_{l}{ }^{\frac{D}{2}}\left(a_{l}{ }^{\frac{1-D}{2}}-a_{c}{ }^{\frac{1-D}{2}}\right)
$$

Given the total normal contact load in a non-dimensional form as

$$
\begin{aligned}
& P^{*}=\frac{D}{2} \psi^{(2-D) / 2}\left(\frac{2(2-D)}{D \psi^{(2-D) / 2}} A_{r}^{*}\right)^{D / 2} \cdot \\
& {\left[\frac{2 \sqrt{\pi} G^{*(D-1)}}{3(3-2 D)}\left(\left(\frac{2(2-D)}{D \psi^{(2-D) / 2}} A_{r}^{*}\right)^{(3-2 D) / 2}-\left(2 a_{c}^{*}\right)^{(3-2 D) / 2}\right)+\right.} \\
& \left.\frac{5.6 \phi \cdot 76.4^{(2-D) / 2(1-D)}\left(2 a_{c}^{*}\right)^{(2-D) / 2}}{2-D}+\frac{2.8\left(1-76.4^{(2.38-1.88 D) /(1-D)}\right) k \phi\left(2 a_{c}^{*}\right)^{(2-D) / 2}}{3(2.38-1.88 D)}\right]
\end{aligned}
$$

where $P^{*}=\frac{P}{E A_{a}} \quad G^{*}=\frac{G}{\sqrt{A_{a}}} \quad A_{r}{ }^{*}=\frac{A_{r}}{A_{a}} \quad a_{c}{ }^{*}=\frac{a_{c}}{A_{a}} \quad \phi=\frac{Y}{E}, \phi$ is plasticity index.

Assuming that the substrate quality of rough surface is $M$, so the damping coefficient is

$$
C_{n}=\eta \sqrt{M K_{n}}=\frac{15 k \sigma_{y}(5-3 D) a_{c}{ }^{\prime 2-D}}{2 E^{*} G^{D-1}(2-D)\left(a_{l}{ }^{\frac{5-3 D}{2}}-a_{c}^{{ }^{\frac{5-3 D}{2}}}\right)} g(D)
$$

where $g(D)=\sqrt{M \frac{E^{*} D}{\sqrt{\pi}(1-D)} \psi^{\frac{2-D}{2}} a_{l} \frac{D}{12}\left(a_{l^{\prime}}{ }^{\frac{1-D}{2}}-a_{c}{ }^{\frac{1-D}{2}}\right)}$

According to formulas above, we can get the dimensionless normal contact damping 


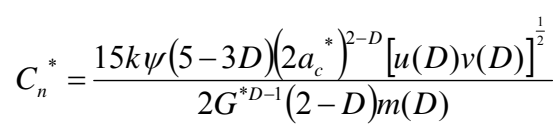

where $C_{n}^{*}=\frac{C_{n}}{A_{a}^{\frac{1}{4}} \sqrt{M E^{*}}} \quad G^{*}=\frac{G}{A_{a}^{\frac{1}{2}}} \quad A_{r}^{*}=\frac{A_{r}}{A_{a}} \quad a_{c}^{*}=\frac{a_{c}}{A_{a}} \quad a_{l}^{\prime}=\frac{2(2-D)}{D \psi^{\frac{2-D}{2}}} A_{r} \quad \psi=\frac{\sigma_{y}}{E^{*}}$

$v(D)=\frac{D}{\sqrt{\pi(1-D)}} \psi^{\frac{2-D}{2}}\left(\frac{2(2-D)}{D \psi^{\frac{2-D}{2}}} A_{r}^{*}\right)^{\frac{D}{2}} \quad u(D)=\left(\frac{2(2-D)}{D \psi^{\frac{2-D}{2}}} A_{r}^{*}\right)^{\frac{1-D}{2}}-\left(2 a_{c}^{*}\right)^{\frac{1-D}{2}}$

$m(D)=\left(\frac{2(2-D)}{D \psi^{\frac{2-D}{2}}} A_{r}^{*}\right)^{\frac{5-3 D}{2}}-\left(2 a_{c}^{*}\right)^{\frac{5-3 D}{2}}$

$A_{a}$ is nominal contact area, $A_{r}{ }^{*}$ is dimensionless real contact area, $G^{*}$ is dimensionless characteristic length scale parameter.

\section{Numerical Simulation of Contact Damping}

From formula (10), (12), given a fixed dimensionless real contact area $A r^{*}, \varphi=1.0$, $H=9 \mathrm{GPa}, E^{*}=130 \mathrm{GPa}, G^{*}$ is $10^{-9}, 10^{-10}, 10^{-11}$ respectively, fractal dimensions are obtained. Simulation results are shown in the Figs:
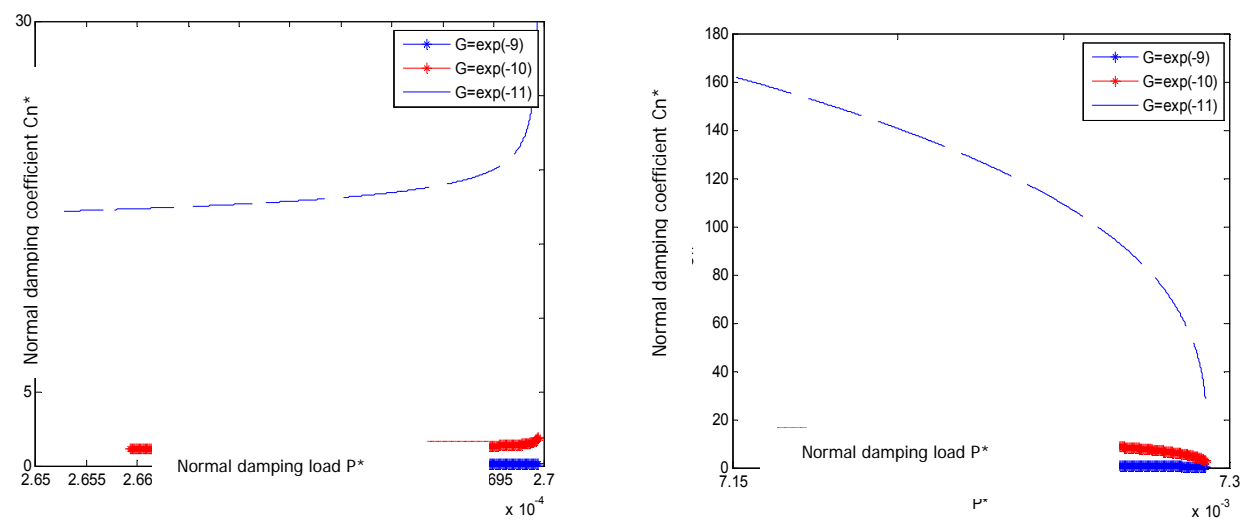

Fig.1 Relationship between load $P^{*}$ and damping $C_{n}^{*}$ as $D=1.4$ and $D=1.6$

From Fig. 1 and Fig.2, we can see that there is an apparent concave nonlinear relation between normal damping and load on two spheres' joint interfaces, when fractal dimension is 1.4. Normal damping increases with normal load. Instead, its relation is convex nonlinear, when fractal dimension is 1.6. It decreases with the increasing of normal load. When fractal dimension is 1.4, normal damping decreases with the increasing of fractal characteristic length parameters or domain extension factor, when fractal dimension is 1.6, it increases with fractal characteristic length parameters or domain extension factor. 

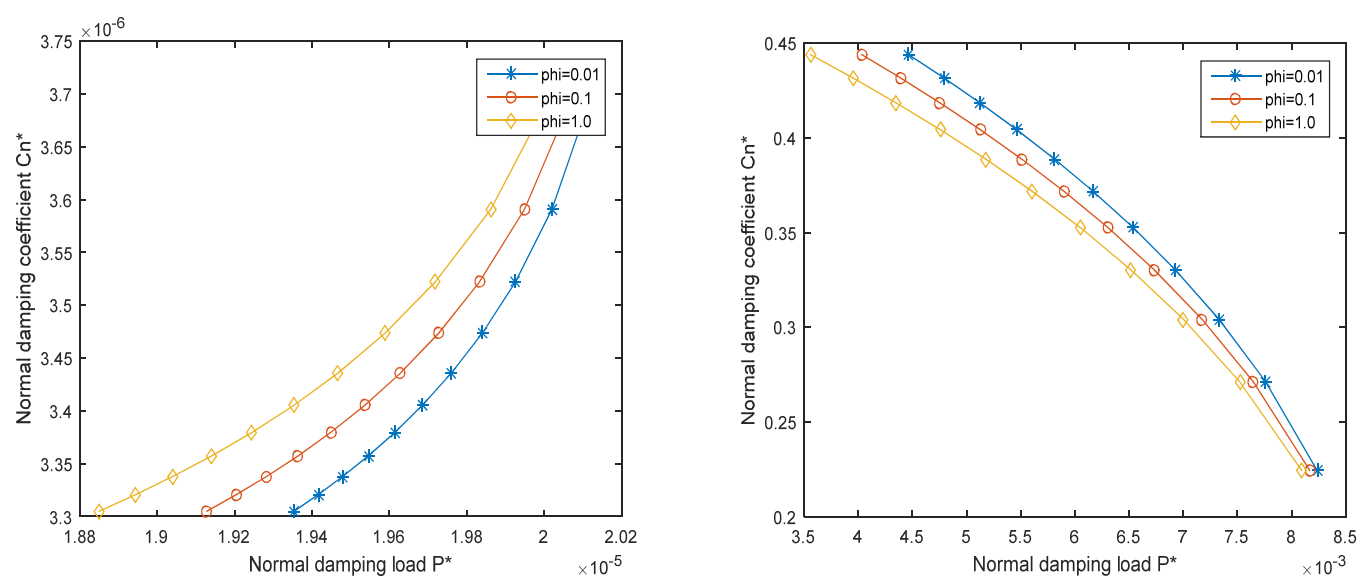

Fig.2 Relationship between load $P^{*}$ and damping $C_{n}^{*}$ as $D=1.4$ and $D=1.6$

\section{Conclusions}

(1) The contact problem of two contact surfaces is simplified to contact problem on two spheres with equal radius. Compared to a rough surface and a rigid planar contact model, it is more universal.

(2) There is an apparent concave nonlinear relation between normal damping and load on two spheres' joint interfaces, when fractal dimension is 1.4. Normal damping increases with normal load. Instead, its relation is convex nonlinear, when fractal dimension is 1.6. It decreases with the increasing of normal load. When fractal dimension is 1.4, normal damping decreases with the increasing of fractal characteristic length parameters or domain extension factor, when fractal dimension is 1.6, it increases with fractal characteristic length parameters or domain extension factor.

\section{Acknowledgements}

This work was financially supported by National university students innovation project (201410504066). Thanks to Prof. Jiajun Yang, he is the corresponding author.

\section{References}

[1] Liu Geng, Liu Tianxiang, Wen Shizhu, Progress in micro nano scale calculation method of contact problems, J. Advances in Mechanics. 38(5)(2008): 521-544.

[2] Li XP, Yue B, Zhao GH, Sun DH, Fractal Prediction Model for Normal Contact Damping of Joint Surfaces considering Friction Factors and Its Simulation. J. Advances in Mechanical Engineering. 2014(2)(2014):1-5.

[3] Zhang Xueliang, Ding Hongqin, Lan Guosheng, Wen Shuhua, Zhang Zongyang, Wang Nanshan, Normal Contact Damping and Dissipation Factor Model of Joint Interfaces Based on Fractal Theory, J. Transactions of the Chinese Society of Agricultural Machinery. (2013): 287-294.

[4] Greenwood J A, Williamson J B P, Contact of nominally flat surfaces, J. Pro. R. Soc.London. 295(1442)(1966):300-319.

[5] Abbott E J, Firestone F A, Specifying surface quality-a method based on accurate measurement and comparison, J. Mechanical Engineers. 55(1933): 569-572.

[6] Chang W R, Etsion I, Bogy D B, An elastic-plastic model for the contact of rough surfaces, J. ASME Journal of Tribology. 109(1987):257-263.

[7] Zhao Yong-wu, L Yan-ming, Jiang Jian-zhong, New elastic-plastic model for the contact of rough surfaces, J. Chinese Journal of Mechanical Engineering. 43(3)(2007):95-101

[8] Wang S, komvopoulos K, A fractal theory of the interfacial temperature distribution in the slow 
sliding regime: part II-multiple domains, elastoplastic contacts and applications, J. Journal of Tribology, Transcations of ASME. 116(4)(1994):824-832. 